\title{
Efficient Implementation of Regulated Morphological Operations Based on Directional Interval Coding ${ }^{\star}$
}

\author{
Gady Agam and Its'hak Dinstein \\ Department of Electrical and Computer Engineering \\ Ben-Gurion University of the Negev \\ Beer-Sheva 84105, Israel
}

\begin{abstract}
The problem of efficient implementation of regulated morphological operations on a serial computer is discussed, where it is assumed that arbitrary kernels may be used. The proposed approach is based on a compact representation of the image which is obtained by using directional interval coding, where the direction of the intervals is selected based on the image contents. This representation is particularly suitable for the processing of directional edge planes. By using stacked intervals, it is described how to obtain the result of a convolution which is based on interval coding, and thereby how to obtain an efficient implementation of regulated morphological operations.
\end{abstract}

Keywords: Mathematical morphology, regulated morphology, directional decomposition, directional interval coding, graphics recognition, document analysis.

\section{Introduction}

Interval coding of binary images is simple and efficient, and is therefore used by many commercial standards such as the CCITT standards. A basic approach for the efficient processing of an image represented by interval coding is described in [1]. The algorithms in that paper are based on an indexing approach of the image elements, and include basic logical operations between two images, and basic morphological operations that use either a four or an eight connected kernel. The algorithm in [2] extends the method described in [1] to arbitrary kernels. However, it does not follow the indexing approach of [1], and so it does not support efficient implementation of basic logical operations between two images.

An efficient implementation of morphological operations, which is based on directional interval coding, has been proposed in $[3,4]$. The implementation in [4] is based on linked lists of directional intervals, which are obtained along four basic directions. This implementation is limited to basic morphological operations with

\footnotetext{
* This work was partially supported by The Paul Ivanier Center for Robotics and Production Automation, Ben-Gurion University of the Negev, Beer-Sheva 84105, Israel.
} 
a $3 \times 3$ kernel, and does not include efficient implementation of basic logical operations. The implementation in [3] extends the method of [1] to support directional interval coding and morphological operations with arbitrary kernels. The extended algorithms preserve the indexing approach of [1], and so efficient implementation of basic logical operations between any number of images may be obtained. In this paper the approach of [3] is extended further to support the efficient implementation of regulated morphological operations.

The following sections discuss the proposed approach in greater detail. Section 2 reviews the regulated morphological operations, and presents some of their relevant properties. Section 3 defines directional interval coding in an arbitrary direction. Section 4 presents algorithms for regulated morphological processing of images which are represented by directional intervals. The summary in Sect. 5 concludes the paper and provides evaluation of the computational complexity of the proposed approach.

\section{Regulated Morphological Operations}

Considering the fitting interpretation of the binary morphological erosion and dilation operations [5], it is possible to observe that they are based on opposing strict approaches. The binary dilation collects shifts for which the kernel set intersects the object set without taking into account what is the size of the intersection, whereas the binary erosion collects shifts for which the kernel set is completely contained within the object set without considering shifts for which some kernel elements are not contained within the object set. As a result of these strict approaches, the ordinary morphological operations are sensitive to noise and small intrusions or protrusions on the boundary of shapes. In order to solve this problem, regulated morphological operations are defined in $[6,7]$ by extending the fitting interpretation of the ordinary morphological operations.

Definition 1. The regulated dilation and erosion of the set $A$ by the kernel set $B$ with a strictness of $s$ is defined respectively by:

$$
\begin{aligned}
& A \dot{\oplus} B \equiv\left\{x \mid \#\left(A \cap(\breve{B})_{x}\right) \geq s\right\} ; s \in[1, \min (\# A, \# B)] \\
& A \stackrel{s}{\ominus} B \equiv\left\{x \mid \#\left(A^{c} \cap(B)_{x}\right)<s\right\} ; s \in[1, \# B]
\end{aligned}
$$

where \#A denotes the cardinality of the set $A, \breve{B}$ denotes the reflection of the set $B$ defined by $\breve{B} \equiv\{x \mid \exists b \in B: x=-b\}$, and $(B)_{x}$ denotes a shift of $B$ by $x$ defined by $(B)_{x} \equiv\{y \mid \exists b \in B: y=x+b\}$.

Proposition 2. The regulated dilation and erosion may be obtained from each other by reflecting the kernel set and complementing the strictness relative to the kernel set:

$$
A \stackrel{s}{\ominus} B=A \stackrel{\overline{s_{B}}}{\oplus B}
$$

where the complement of the strictness $s$ relative to the set $B$ is defined by: $\overline{s_{B}} \equiv \# B-s+1$. 
Proposition 3. Given two sets $A, B \subset \mathcal{Z}^{2}$, the regulated dilation and erosion of $A$ by $B$ may be obtained by thresholding the linear convolution between their respective binary images $\underline{A}$ and $\underline{B}$ :

$$
\begin{aligned}
& A \stackrel{s}{\oplus} B=\{(k, l) \mid \underline{A}(k, l) * \underline{B}(k, l) \geq s\} \\
& A \stackrel{s}{\ominus} B=\left\{(k, l) \mid \underline{A^{c}}(k, l) * \underline{\breve{B}}(k, l)<s\right\}
\end{aligned}
$$

where $*$ denotes linear convolution.

Based on the basic regulated morphological operations it is possible to define an extensive regulated dilation and an anti-extensive regulated erosion. By using the extensive operations it is possible to define idempotent regulated open and close operations which extend the fitting interpretation of the ordinary open and close operations. Further properties of the regulated morphological operations are discussed in $[6,7]$, and it is shown that they possess many of the properties of the ordinary morphological operations. In particular it is shown that the ordinary morphological operations may be obtained as special cases of the regulated morphological operations when using a strictness of one.

\section{Directional Interval Coding}

Given an $M \times N$ image, it is possible to scan it in an arbitrary direction $\phi$ along parallel discrete straight line segments so that each element of the image is covered by a single scan line. In the proposed approach for directional scanning of the image, uniform length scan lines are used, and so elements on neighboring scan lines may be obtained in a simple way. In order to get uniform scan lines the image is zero padded appropriately. Since the scanning of an image in the direction of $\theta$ is essentially the same as scanning in the direction of $\theta+180^{\circ}$, the discussion in this paper is limited to the angular range of $\left[-135^{\circ}, 45^{\circ}\right)$.

As described in [3], the starting point of the $l$-th scan line may be obtained by:

$$
P_{s}(l)=\left\{\begin{array}{l}
(0, l-\max (0, \Delta j)) \text { if } \theta \in[-135,-45) \\
(l-\max (0, \Delta i), 0) \text { if } \theta \in[-45,45)
\end{array}\right.
$$

where $(\Delta i, \Delta j)$ is the difference between the ending and starting points of a scan line. The offset of the $c$-th element on a scan line may be evaluated by:

$$
P_{d}(c)=\left\{\begin{array}{l}
(c,\lfloor-c \cdot \cot (\theta)+0.5\rfloor) \text { if } \theta \in[-135,-45) \\
(\lfloor-c \cdot \tan (\theta)+0.5], c) \text { if } \theta \in[-45,45)
\end{array}\right.
$$

Based on (6) and (7), the point that corresponds to the $c$-th column on the $l$-th scan line, is given by: $P(l, c)=P_{s}(l)+P_{d}(c)$. Since the length of each scan line is uniform, it is possible to assign a unique index to a point $P(l, c)$, which is the image point that is covered by the $c$-th column of the $l$-th scan line, by: $I_{M, N, \theta}(P(l, c)) \equiv l \cdot N_{\theta}+c$, where $c \in\left[0, N_{\theta}-1\right], l \in\left[0, M_{\theta}-1\right]$, and $M_{\theta}$ and $N_{\theta}$ denote the number of scan lines and scan columns respectively. 
When the image elements are represented by indexes, it is possible to specify an interval by using its starting index $I_{b}$ and its ending index $I_{e}$. Since in the proposed approach it is required to distinguish between the starting and ending indexes of an interval, modified indexes that are based on $I_{b}$ and $I_{e}$ are used for representing the interval. The starting index of an interval is defined as an even index given by $b=2 \cdot I_{b}$, whereas the ending index of an interval is defined as an odd index given by $e=2 \cdot I_{e}+1$. Consequently, the interval $R=(b, e)$ is an interval that begins at the image element with an index of $\lfloor b / 2\rfloor$ and ends at the image element with an index of $\lfloor e / 2\rfloor$. The number of elements that are covered by the interval $R=(b, e)$ is given by: $\|R\|=(e-b+1) / 2$.

Given a binary image $\underline{A}$ it is possible to scan it and construct a set $A$ containing all the intervals in the image $A$. By using the set of intervals $A$, the image $\underline{A}$ may be obtained by $\bigcup_{R_{a} \in A} R_{a}$. Conversely, given a set of intervals $A$ that represents an $M \times N$ image, it is possible to reconstruct the image $A$ by scanning an empty $M \times N$ image in the direction of the intervals, and setting all the elements $(i, j)=P(l, c)$ that belong to an interval provided that $i \in[0, M-1]$ and $j \in[0, N-1]$. A situation in which an interval is not contained completely inside the image area may occur after processing the set of intervals.

\section{Morphological Processing of Intervals}

This section discusses how the intervals of the result of a regulated dilation and erosion may be obtained by using the sets of intervals that represent the kernel and the original image. As explained later, the proposed implementation uses shifted intervals. In order to have a uniform rule for determining a required shift value which does not depend on the relative location on the scan line, and in order to prevent the breaking of intervals into several intervals when shifting them, uniform scan lines have to be used. Uniform scan lines are obtained in directions of: $-135^{\circ},-90^{\circ},-45^{\circ}$, and $0^{\circ}$. As shown later, uniform scan lines contribute further to the efficiency of the operations by reducing the number of required shifts. Based on the above description, the discussion in this section is limited to intervals which are obtained by using uniform scan lines.

\subsection{The Intervals of a Convolution}

The proposed efficient implementation of the regulated dilation and erosion operations is based on their linear filtering interpretation. Therefore, this section describes an efficient implementation of the convolution operation.

Based on the linearity of the convolution operation, we get that the convolution between two unions of intervals is equivalent to the union of the convolution between the individual intervals. That is: $\left(\bigcup_{R_{a} \in A} R_{a}\right) *\left(\bigcup_{R_{b} \in B} R_{b}\right)=$ $\bigcup_{R_{a} \in A} \bigcup_{R_{b} \in B}\left(R_{a} * R_{b}\right)$. Therefore, the convolution between the image and the kernel set of intervals may be obtained by processing the individual intervals separately. The convolution between two intervals results in a longer interval. However, the values along the obtained interval are not uniform, and so this 
interval may not be specified completely by using only its starting and ending indexes. In order to be able to represent the result of the convolution between two intervals by using uniform valued intervals, which are specified by their starting and ending indexes, it is possible to decompose the result of the convolution into a sum of uniform valued intervals. That is, the result of the convolution may be represented by stacking several uniform valued intervals.

Consider the convolution between an interval $R_{a}$ and a shorter interval $R_{b}$. Based on the definitions of convolution and ordinary dilation, it is possible to observe that the size of the resulting non-uniform interval is given by $\left\|R_{a} \oplus R_{b}\right\|$. The leftmost and rightmost elements in the resulting interval have a value of one, the second leftmost and the second rightmost elements have a value of two, and so on. The maximal value in the resulting interval is given by $\left\|R_{b}\right\|$. Since the convolution operation is commutative, the case in which $R_{a}$ is shorter than $R_{b}$ is handled similarly, where the maximal value in the resulting interval is given in this case by $\left\|R_{a}\right\|$. Based on the above description, it is possible to conclude that the set of stacked intervals required for representing the result of the convolution between the intervals $R_{a}$ and $R_{b}$, has $\min \left(\left\|R_{a}\right\|,\left\|R_{b}\right\|\right)$ intervals. The largest interval is given by $R_{a} \oplus R_{b}$, and each consequent interval is shorter by one from each side.

The evaluation of the dilation of the interval $R_{a}$ by the interval $R_{b}$, may be obtained by considering the interval $R_{b}$ as describing a set of shifts, which is used by the dilation operation for generating a union of shifts of the interval $R_{a}$. In order to be able to use the interval $R_{b}$ as describing a set of shifts, the length of a scan line in $A$ and $B$ should be identical. Therefore, when assuming that the image is of size $M \times N$, the kernel is of size $P \times Q$, and that the kernel is smaller than the image $(P<M, Q<N)$, the kernel should be enlarged to a size of $M \times N$. This enlargement may be obtained by using the set of intervals $B$, as described in [3]. After the required enlargement, the kernel should be centered around its origin. When denoting the origin of the kernel by the point $P_{o}$, the index of $P_{o}$ in the enlarged kernel is given by $I_{M, N, \theta}\left(P_{o}\right)$, and so the intervals of the enlarged kernel should be centered by subtracting $2 \cdot I_{M, N, \theta}\left(P_{o}\right)$ from their vertices. In the following discussion we assume that $B^{\prime}$ represents the intervals of the enlarged and centered kernel, and that $R_{b} \in B^{\prime}$.

Consider the dilation of the interval $R_{a}=\left(s_{a}, e_{a}\right)$ by the interval $R_{b}=\left(s_{b}, e_{b}\right)$ which is obtained by taking the union of all the shifts of $R_{a}$ which belong to $R_{b}$. Based on the fact that $R_{a}$ and $R_{b}$ are intervals that belong to a uniform scan line, it is possible to use only the two most extreme shifts specified by $R_{b}$, which are the vertices of that interval. In that way, the resulting interval may be obtained by taking the starting index of the leftmost shift of $R_{a}$, and taking the ending index of the rightmost shift of $R_{a}$. Therefore, we obtain that $R_{a} \oplus R_{b}=\left(s_{a}+s_{b}, e_{a}+e_{b}\right)-(0,1)=R_{a}+R_{b}-(0,1)$, where the subtraction of $(0,1)$ is required in order to get an odd ending index.

By using the evaluation of $R_{a} \oplus R_{b}$, the required set of stacked intervals that 
represents the convolution between the intervals $R_{a}$ and $R_{b}$ is given by:

$$
\begin{aligned}
& \left\{R_{a} * R_{b}\right\}=\left\{(x+2 \cdot(i-1), y-2 \cdot(i-1)) \mid(x, y)=R_{a}+R_{b}-(0,1),\right. \\
& \left.\quad 1 \leq i \leq \min \left(\left\|R_{a}\right\|,\left\|R_{b}\right\|\right)\right\}
\end{aligned}
$$

By using (8), the set of stacked intervals that represents the result of the convolution between $\underline{A}$ and $\underline{B}$, may be evaluated by:

$$
\mathcal{C}(A, B)=\left\{\left\{R_{a} * R_{b}\right\} \mid R_{a} \in A, R_{b} \in B^{\prime}\right\}
$$

where $B^{\prime}$ is the enlarged and centered kernel.

\subsection{The Intervals of a Regulated Dilation}

Based on (4) and the construction of the convolution $\mathcal{C}(A, B)$, the result of the regulated dilation of $A$ by $B$ with a strictness of $s$ may be obtained by locating parts of intervals that are covered by at list $s$ intervals of the convolution $\mathcal{C}(A, B)$. In order to determine the number of intervals that cover each image point, it is possible to construct an ordered vector $\bar{X}$, containing all the vertices of all the intervals in $\mathcal{C}(A, B)$, sorted in an increasing order. By scanning this vector from beginning to end, it is possible to construct a counter that starts with a value of zero, increased by one when the beginning of an interval is reached (an even index), and decreased by one when the end of an interval is reached (an odd index). As demonstrated in [3], the number of intervals that contain the $k$-th element of the vector $\bar{X}$ (which is denoted by $x_{k}$ ) is given by: $C(\bar{X}, k)=k-2 \cdot \sum_{i=1}^{k}\left(x_{i} \bmod 2\right)$.

By using the counter $C(\bar{X}, k)$, it is possible to obtain the intervals of the regulated dilation with a strictness of $s$, by scanning the ordered vector $\bar{X}$ from beginning to end, and looking for intervals that begin at an element $x_{i}$ for which $C(\bar{X}, i)=s$ and end at the first element $x_{j}$ after $x_{i}$ for which $C(\bar{X}, j)=s-1$. When scanning $\bar{X}$ from beginning to end, the value of $C(\bar{X}, i)=s$ is obtained at the beginning of an interval, and so $x_{i}$ is an even index, while the value of $C(\bar{X}, j)=s-1$ is obtained at the end of an interval, and so $x_{j}$ is an odd index. Hence, the set of intervals that represents the image obtained by the regulated dilation of $\underline{A}$ by $\underline{B}$ with a strictness of $s$, may be evaluated by:

$$
\begin{aligned}
& \mathcal{D}^{s}(A, B)=\left\{\left(x_{i}, x_{j}\right) \mid x_{i}, x_{j} \in \bar{X}, i<j, C(\bar{X}, i-1)=s-1,\right. \\
&C(\bar{X}, i)=s, C(\bar{X}, j)=s-1, \forall k \in[i+1, j-1]: C(\bar{X}, k) \geq s\}
\end{aligned}
$$

where it is assumed that $C(\bar{X}, 0) \equiv 0$.

\subsection{The Intervals of a Regulated Erosion}

An efficient implementation of the regulated erosion, which is based on the processing of intervals, may be obtained by using the complement of the image and the reflection of the kernel in (5) in a way similar to the one described for the regulated dilation. However, since in an ordinary line-drawing image the number 
of intervals in a complemented image is larger than the number of intervals in the original image, the obtained implementation will be less efficient. Alternatively, when using (3), it is possible to obtain a more efficient implementation of the regulated erosion by:

$$
\mathcal{E}^{s}(A, B)=\overline{\mathcal{D}^{\bar{s}}}(A, \breve{B})
$$

In order to evaluate the regulated erosion $\mathcal{E}^{s}(A, B)$ by (11), the convolution $\mathcal{C}(A, \breve{B})$ should be obtained. Based on the definition of reflection, the index of a reflected element is obtained by inverting the sign of the original index. When reflecting an interval $R_{b}$, in addition to the sign inversion of the starting and ending indexes, the reflection causes the starting index to become the ending index of the reflected interval, and the ending index to become the starting index of the reflected interval. When exchanging the starting and ending inciexes of an interval, in order to obtain its reflection, the starting and ending indexes of the reflected interval should be converted to be even and odd respectively. Therefore, the ending index of the original interval should be decreased by one (before inverting its sign), and the starting index of the original interval should be increased by one (after inverting its sign). Following the above description, we get that the reflection of an interval $R_{b}$ is given by $\breve{R}_{b}=\left(-e_{b}+1,-s_{b}+1\right)$, and so the intervals of the reflected kernel are given by: $B^{\prime \prime}=\left\{\left(-e_{b}+1,-s_{b}+1\right) \mid\left(s_{b}, e_{b}\right) \in\right.$ $B^{\prime}$. The convolution $\mathcal{C}(A, \breve{B})$ may be obtained by using $B^{\prime \prime}$ instead of $B^{\prime}$ in (9).

\section{Summary}

The table in Fig. 1 summarizes the computational complexity evaluation of the proposed operations, where a detailed description of this evaluation may be found in [8]. In this table it is assumed that the image is in the size of $M \times M$, the kernel is in the size of $P \times P$, and that the number of intervals in the image and the kernel is $m$ and $p$ respectively. In the evaluation of the basic logical operations, it is assumed that there are $n$ images.

The ratio between $m$ and $M$, and between $p$ and $P$, depends on the contents of the image. When considering the processing of ordinary maps and line-drawing images, it is possible to assume that $m$ and $M$ have the same order of magnitude, and that $p$ and $P$ have the same order of magnitude. Since in most applications $P \ll M$ and $n \ll M$, in such a case we get that the computational complexity of a straightforward implementation reduces to $\mathbf{O}\left(M^{2}\right)$, whereas the computational complexity of the proposed approach reduces to $\mathbf{O}(m)$. It should be noted that in addition to its reduced computational complexity, the proposed approach reduces significantly the memory requirements of the application. When processing large maps/documents, which were scanned with a fine resolution, in several different color layers, a reduction in the memory requirements of the application is essential. This reduction recomes even more important when considering the directional decomposition of each image into several directional edge planes as proposed in $[9,10]$. 


\begin{tabular}{|c|c|c|c|c|c|}
\hline & (a) & (b) & (c) & (d) & (e) \\
\hline & $\begin{array}{c}\text { Intersection } \\
\text { Union } \\
\text { XOR }\end{array}$ & Complement & $\begin{array}{c}\text { Dilation } \\
\text { Erosion } \\
\text { (Ordinary) }\end{array}$ & Convolution & $\begin{array}{c}\text { Dilation } \\
\text { Erosion } \\
\text { (Regulated) }\end{array}$ \\
\hline $\begin{array}{l}\text { Straightforward } \\
\text { Implementation }\end{array}$ & $\mathrm{O}\left(n M^{2}\right)$ & $\mathrm{O}\left(M^{2}\right)$ & $\mathbf{O}\left(P^{2} M^{2}\right)$ & $\mathrm{O}\left(P^{2} M^{2}\right)$ & $\mathrm{O}\left(P^{2} M^{2}\right)$ \\
\hline $\begin{array}{l}\text { Efficient } \\
\text { Implementation }\end{array}$ & $\mathrm{O}(n m \log n)$ & $\mathrm{O}\left(m+M_{\theta}\right)$ & $\mathrm{O}(p m \log p)$ & $\mathbf{O}(p P m)$ & $\mathbf{O}(p P m \log p P)$ \\
\hline
\end{tabular}

Fig. 1. Summary of the computational complexity of the proposed operations.

\section{References}

1. I. T. Young, R. L. Peverini, P. W. Verbeek, and P. J. Van-Otterloo, "A new implementation for the binary and Minkowski operators", Computer Graphics and Image Processing, Vol. 17, No. 3, pp. 189-210, 1981.

2. L. Ji, J. Piper, and J. Y. Tang, "Erosion and dilation of binary images by arbitrary structuring elements using interval coding", Pattern Recognition Letters, Vol. 9, pp. 201-209, 1989.

3. G. Agam, J. Frydman, O. Amiram, and I. Dinstein, "Efficient morphological processing of maps and line-drawings based on directional interval coding", in Vision Geometry VI, R. A. Melter, A. Y. Wu, L. J. Latecki eds., Proc. SPIE 3168, pp. 41-51, July, 1997.

4. H. Luo and R. Kasturi, "Improved directional morphological operations for separation of characters from maps/graphics", in Proc. GREC'97, Nancy, France, pp. 8-15, August, 1997.

5. R. M. Haralick, S. R. Sternberg, and X. Zhuang, "Image analysis using mathematical morphology", IEEE Trans. PAMI, Vol. 9, No. 4, pp. 532-550, 1987.

6. G. Agam and I. Dinstein, "Generalized morphological operators applied to map-analysis", in Advances in Structural and Syntactical Pattern Recognition, P. Perner, P. Wang, A. Rosenfeld eds., LNCS Vol. 1121, pp. 60-69, 1996.

7. G. Agam and I. Dinstein, "Regulated morphological operations", Submitted for publication.

8. G. Agam and I. Dinstein, "Efficient implementation of directional and regulated morphological operations", Submitted for publication.

9. H. Yamada, K. Yamamoto, and K. Hosokawa, "Directional mathematical morphology and reformalized Hough transformation for the analysis of topographic maps", IEEE Trans. PAMI, Vol. 15, No. 4, pp. 380-387, 1993.

10. G. Agam and I. Dinstein, "Directional decomposition of line-drawing images based on regulated morphological operations", in Graphics Recognition: Algorithms and Systems, K. Tombre, A. K. Chhabra eds., LNCS Vol. 1389, pp. 21-34, 1998. 